\title{
Analistas simbólicos, jerarquía urbana y atracción de talento en las ciudades mundiales españolas (2005-2019): un análisis a partir de la EPA*
}

\author{
Juan Miguel Albertos Puebla \\ Universitat de València. Departamento de Geografía \\ Institut Interuniversitari de Desenvolupament Local \\ juan.m.albertos@uv.es
}

\section{Resumen}

Este trabajo utiliza los microdatos de la Encuesta de población activa del INE entre 2005 y 2019 para analizar el nivel de competencias de la población ocupada, los procesos de cualificación del mercado de trabajo de las ciudades mundiales españolas (Madrid, Barcelona, Valencia, Sevilla y Bilbao) y el papel que están teniendo las migraciones de profesionales y técnicos altamente cualificados como reflejo de su capacidad diferencial para atraer talento externo. Siguiendo el marco conceptual propuesto por Allen J. Scott de creciente polarización de los mercados de trabajo metropolitanos entre una clase de "analistas simbólicos» de alta cualificación y una «subclase de servicios», se considerarán los efectos que han tenido la profunda crisis que se inicia en 2009 y la recuperación subsiguiente hasta 2019 en las tendencias de cambio hacia una red urbana menos primada y más heterárquica.

Palabras clave: ciudades globales; segundo escalón metropolitano; jerarquía urbana; trabajadores del conocimiento; atracción de talento; Encuesta de población activa

* Este artículo se ha elaborado en el marco del proyecto «Sostenibilidad social, conectividad global y economía creativa como estrategias de desarrollo en el Área Metropolitana de Valencia» (CSO2016-74888-C4-1-R), financiado por la Agencia Estatal de Investigación (AEI) y el Fondo Europeo de Desarrollo Regional (FEDER) dentro del Programa Estatal de Investigación, Desarrollo e Innovación Orientada a los Retos de la Sociedad, incluido en el Plan Estatal de Investigación Científica y Técnica y de Innovación 2013-2016, convocatoria de 2016. 
Resum. Analistes simbòlics, jerarquia urbana y atracció de talent a les ciutats mundials espanyoles (2005-2019): una anàlisi a partir de l'EPA

Aquest treball utilitza les microdades de l'Enquesta de població activa de l'INE entre 2005 i 2019 per analitzar el nivell de competències de la població ocupada, els processos de qualificació del mercat de treball de les ciutats mundials espanyoles (Madrid, Barcelona, València, Sevilla i Bilbao) i el paper que estan tenint les migracions de professionals i tècnics altament qualificats com a reflex de la seva capacitat diferencial per atreure talent extern. Seguint el marc conceptual proposat per Allen J. Scott de creixent polarització dels mercats de treball metropolitans entre una classe d' "analistes simbòlics» d'alta qualificació i una "subclasse de serveis", es consideraran els efectes que han tingut la profunda crisi que es va iniciar en 2009 i la recuperació subsegüent fins a 2019 en les tendències de canvi cap a una xarxa urbana menys privilegiada i més heteràrquica.

Paraules clau: ciutats globals; segon esglaó metropolità; jerarquia urbana; treballadors de el coneixement; atracció de talent; Enquesta de població activa

Résumé. Analystes symboliques, hiérarchie urbaine et attraction des talents dans les villes mondiales espagnoles (2005-2019)

Ce travail utilise les microdonnées de l'enquête sur la population active de l'INE entre 2005 et 2019 pour analyser le niveau de compétences de la population employée et les processus de qualification du marché du travail dans les villes-monde espagnoles (Madrid, Barcelone, Valence, Séville et Bilbao) et le rôle que jouent les migrations de professionnels et de techniciens hautement qualifiés comme reflet de leur capacité différentielle à attirer des talents externes. En suivant le cadre conceptuel proposé par Allen J. Scott d'augmentation de la polarisation des marchés du travail métropolitains entre une classe 'd'analystes symboliques' hautement qualifiés et une 'sous-classe de services', on analysera les effets de la crise profonde qui commence en 2009 et la reprise qui s'ensuit jusqu'en 2019 dans les tendances d'évolution vers un réseau urbain moins privilégié et plus hétérarquique.

Mots-clés : villes mondiales ; deuxième étape métropolitaine ; hiérarchie urbaine ; travailleurs du savoir ; attraction des talents ; Enquête Emploi

Abstract. Symbolic analysts, urban hierarchy, and talent attraction in Spanish world cities (2005-2019)

This work uses microdata from the National Institute of Statistics' Labor Force Survey between 2005 and 2019 to analyze the level of competencies of the employed population and the qualification processes of the labor market in Spanish world cities (Madrid, Barcelona, Valencia, Seville, and Bilbao) and the role that migrations of highly qualified professionals and technicians are playing as a reflection of their differential capacity to attract external talent. Following the conceptual framework proposed by Allen J. Scott of increasing polarization of metropolitan labor markets between a class of highly qualified "symbolic analysts" and a "services subclass," the effects of the deep crisis beginning in 2009 until the subsequent recovery until 2019 on trends of change towards a less privileged and more heterarquical urban network will be considered.

Keywords: world cities; second-tier cities; urban hierarchy; knowledge workers; talent attraction; Labor Force Survey 


\section{Sumario}
1. Introducción
4. Resultados y discusión
2. Marco teórico y conceptual
5. Conclusiones
3. Fuentes y metodología
Referencias bibliográficas

\section{Introducción}

Las grandes ciudades, en tanto que polos de innovación y creatividad, son piezas fundamentales para superar los problemas de eficiencia económica y bajo crecimiento de la productividad. Además, al actuar como elementos de articulación entre las economías regionales y locales y la economía global, tienen un impacto territorial que supera el mero ámbito urbano y metropolitano. En este contexto, las transformaciones recientes de las estructuras productivas, laborales y ocupacionales de las ciudades globales españolas — nuestro objeto geográfico de estudio - solo se entienden a partir de su inserción en redes de relaciones multiescalares dentro de las cuales cumplen funciones específicas. Como "ciudades-región en un mosaico global» (Scott, 2012: 59), cada una de ellas, en función de sus dotaciones y recursos, va ajustando la forma en que se inserta globalmente y el carácter de sus relaciones con su entorno local/ regional. Esta inserción en el sistema global se concreta en la existencia de redes de relaciones intermetropolitanas a diferentes escalas (nacional, europea, global) sostenidas por flujos materiales e inmateriales (información, capital y personas), de las que depende el potencial desarrollo de sus entornos regionales (Scott, 2012).

El programa ESPON 2013 ha desplegado diversas iniciativas que nos ofrecen un marco adecuado para estas dinámicas. El proyecto TIGER (Territorial Impact of Globalization for Europe and its Regions) concluye que la suerte de las diferentes regiones en un entorno globalizado está en clara relación con su sistema urbano y su capacidad para articular el territorio a múltiples escalas. Así, en España solo considera como regiones con una baja vulnerabilidad a los efectos negativos de la globalización a Madrid, Cataluña, la región compuesta por el País Vasco-Navarra-La Rioja y la Comunidad Valenciana (ESPON, 2012a). En una línea discursiva similar, la iniciativa SGPTD (Secondary Growth Poles and Territorial Development in Europe) trata específicamente el papel de las áreas metropolitanas y ciudades del segundo escalón urbano, lo que en el caso español supone evaluar las potencialidades de Barcelona, Valencia, Sevilla y Bilbao, más allá de la enorme primacía que ha cobrado Madrid. (ESPON, 2012b).

Este artículo quiere profundizar en estas dinámicas en el caso español tomando como objeto de estudio las que podemos considerar más claramente como ciudades globales (Madrid, Barcelona, Valencia, Sevilla y Bilbao), "gateways cities» cuya funcionalidad para liderar el crecimiento y la innovación regional va a depender en buena medida de su capacidad para atraer y generar 
talento y para cualificar su estructura productiva, laboral y ocupacional. El interés por este tema surge además de la constatación de que algunas grandes áreas metropolitanas españolas muestran un nivel funcional claramente inferior al que les correspondería por su tamaño demográfico y económico (Rozenblat, 2007), lo que podría estar afectando negativamente a sus entornos regionales. $\mathrm{Al}$ mismo tiempo, el impacto de la crisis económica iniciada en 2008 parece haber tenido una influencia asimétrica, al desatar procesos de divergencia regional no solo durante la fase de recesión más intensa, sino también en el subsiguiente período de recuperación (Albertos y Sánchez, 2017; Albertos, 2019). Este sería sin duda un claro punto de inflexión respecto a las tendencias previas que desde los años 1980 apuntaban a una diminución de las desigualdades en términos de productividad, renta per cápita y empleo (Hernández y Usabiaga, 2016; Barrios et al., 2017).

De ahí que sea oportuno analizar con algún detalle el papel que están ejerciendo las grandes ciudades españoles como motores de modernización y competitividad, así como los impactos que pueden haberse derivado de la crisis iniciada en 2008. Para ello pondremos el foco del análisis en la estructura ocupacional, en el nivel de cualificación y competencial de su mercado de trabajo, en suma, en su capacidad para atraer y albergar talento. De esta manera, podremos evaluar su nivel funcional y su capacidad para ser verdaderos motores de la modernización y la innovación regional.

\section{Marco teórico y conceptual}

En el tránsito del fordismo al posfordismo, Scott (1988) identificó tres tipos de sectores en los que se hacía especialmente evidente la existencia de procesos de reaglomeración espacial, bien en entornos metropolitanos, bien bajo otras formas territoriales. Estos tres tipos de actividad siguen constituyendo hoy, tres décadas después, la base económica de las regiones más innovadoras y desarrolladas:

a) Sectores de alta tecnología ligados a la industria aeroespacial, la biotecnología y las nuevas tecnologías de la comunicación y la información.

b) Industrias intensivas en trabajo cualificado de bienes de consumo muy ligados a la moda y el diseño que compiten en virtud de su alta calidad y diferenciación.

c) El sector FIRE (finance, inssurance, real estate), que incluye las actividades financieras, los seguros y el sector inmobiliario, a menudo junto con otros servicios avanzados a las empresas, la publicidad, el marketing y los servicios legales. A este sector FIRE, se le suele añadir, recientemente, un sector ICE (intellectual, cultural, educational), que subraya la importancia de la economía de la cultura y en general de las actividades creativas y del conocimiento.

El crecimiento del sector FIRE e ICE está en relación con la acuciante necesidad de generar, intercambiar, gestionar, interpretar y emplear grandes volú- 
menes de información en múltiples ámbitos: incorporación de conocimiento científico, innovación en productos y procesos, obtención de financiación, estrategias de inversión, prospectiva de nuevos mercados, logística, auditoría, asesoría legal, etcétera. Así, resulta apropiado definir la sociedad actual como una sociedad de la información, usando la terminología de Castells (1996) y atendiendo a la importancia que esta adquiere como elemento central de la innovación, la organización y la producción económica.

Los servicios avanzados a la producción, junto con una industria recualificada y los sectores de creación y difusión cultural, componen la actual base servindustrial de la economía de las áreas metropolitanas más avanzadas e innovadoras (Graham y Spence, 1995; Daniels y Bryson, 2002; Méndez, 2006, 2008) de las ciudades donde se concentran las "clases creativas», según el término popularizado por Florida (2002 y 2007).

Más aún, el nuevo escenario productivo, con sus nuevas exigencias de innovación continua, escala global e intensividad en el uso de información y conocimiento, ofrece claras ventajas a los entornos metropolitanos (Scott, 2006; Méndez, 2008), dado que estos pueden aprovechar los recursos específicos generados a través de su trayectoria de desarrollo que resultan críticos en el nuevo escenario: servicios avanzados, recursos humanos cualificados, centros de investigación e I+D y conocimiento en clústeres sectoriales consolidados. $Y$, no menos importante, cuentan con infraestructuras específicas de comunicaciones (aeropuertos internacionales, estaciones de tren de alta velocidad, plataformas logísticas) y de telecomunicaciones (red de fibra óptica, red inalámbrica), que son necesarias para movilizar los flujos materiales (personas, bienes) e inmateriales (información, conocimiento) que dan forma a la economía global (Smith y Timberlake, 1995).

La presencia de estas actividades de alto valor añadido se considera necesaria para asegurar la competitividad de la ciudad metropolitana y conseguir una inserción exitosa en la división espacial del trabajo propia de la nueva economía global. Pero, junto con estas actividades básicas, y como elemento también necesario para esta forma de acumulación, surgen y florecen otra serie de servicios que aseguran el funcionamiento interno de la metrópolis: servicios a la población, distribución comercial, servicios banales a las empresas, trasporte urbano o construcción. Méndez (2006) considera esta dualidad productiva como un hecho consustancial al devenir de las grandes áreas metropolitanas en la globalización, y establece la distinción entre sectores de actividad de alto valor añadido y conexión con el exterior y sectores de actividad ligados al mantenimiento y la reproducción social y material de la metrópolis. Esta dualidad en la estructura económica metropolitana tendría su reflejo en un mercado de trabajo posfordista, crecientemente segmentado y fragmentado en función de la estabilidad en el empleo, las posibilidades de promoción, la cualificación y el sector de encuadre. Esta situación se traduce en una creciente desigualdad social. El aumento de la proporción de los empleos mejor pagados y de más alta cualificación transcurriría en paralelo a la dualización sociolaboral entre un mercado primario y un mercado secundario de trabajo (Méndez, 2006), entre 
«los analistas simbólicos, trabajadores altamente cualificados del conocimiento y la cultura, y la subclase de servicios" (Scott, 2012: 95). Los primeros, con una superior cualificación a menudo de nivel universitario, disfrutan de unas mejores condiciones laborales, estabilidad, y de la posibilidad de desarrollar una carrera profesional, mientras que los segundos sufren unas condiciones laborales peores y una gran precariedad.

La presencia en un área metropolitana/urbana de estos analistas simbólicos, trabajadores de conocimiento altamente cualificados, puede ser utilizada como un indicador de su nivel funcional y de su grado de éxito a la hora de transformar sus estructuras productivas e integrarse con éxito, de forma innovadora y competitiva, en la nueva y emergente economía global (Musterd et al., 2016). De hecho, su localización dentro del sistema urbano muestra una intensa y creciente concentración en las áreas urbanas y metropolitanas de mayor tamaño y jerarquía funcional (Scott, 2012; Escolano y Escalona, 2017). Este proceso se asocia a menudo a la configuración de un nuevo sistema urbano o red de ciudades mundiales, the world city hypothesis (Friedmann, 1986), profundamente interrelacionadas, que coordinan y organizan el funcionamiento de la economía global y que lideran su crecimiento, bien porque concentran las actividades y funciones de mayor valor añadido (Knox y Taylor, 1995; Taylor, 2001, 2003), bien porque en su seno surgen las innovaciones más disruptivas dentro de los sectores financiero y de servicios avanzados (Sassen, 1991, 1994).

No obstante, recientemente se está prestando una gran atención al comportamiento de las ciudades metropolitanas que no son cabecera de sus sistemas urbanos nacionales, las denominadas second tier cities o ciudades de segundo escalón (ESPON, 2012b; Camagni y Capello, 2015; Camagni et al., 2015). Este interés surge de la apreciación de que estas ciudades están teniendo un comportamiento especialmente dinámico y mejor que el de las ciudades cabecera. Si bien este interés no es nuevo (Markusen et al., 1999), su mejor comportamiento solía relacionarse con la presencia de economías de localización o de distrito. En cambio, en la actualidad su mejor trayectoria se asocia al disfrute de economías de aglomeración de naturaleza plenamente urbana, al tiempo que las mayores áreas metropolitanas estarían enfrentando deseconomías de aglomeración. Esta es la tesis propuesta por Djikstra et al. (2013) en su análisis de la evolución reciente del sistema urbano europeo, según la cual el crecimiento económico desde comienzos del nuevo milenio debe más a las ciudades de segundo escalón que a las ciudades cabecera. En un sentido parecido, Camagni et al. (2015) documentan que las grandes ciudades del segundo escalón (entre 250.000 y 1.000 .000 de habitantes) en la EU15 se muestran más resilientes en períodos de recesión, mientras que Parkinson et al. (2015) consideran que el crecimiento agregado nacional se ve fortalecido si se produce una descentralización de responsabilidades, capacidades, recursos e inversiones hacia un rango de ciudades más amplio que si estas se concentran en la ciudad cabecera. Esto sería especialmente necesario si la distancia entre la ciudad cabecera y las de segundo nivel es especialmente grande, si las infraestructuras de las ciudades de segundo nivel son relativamente débiles y si la ciudad cabecera empieza a sufrir deseconomías de aglomeración. 
Aquí el reto está en discernir cómo funcionan las economías de aglomeración en estas grandes ciudades del segundo escalón, pues no todas las ciudades de este tamaño las disfrutan (Camagni y Capello, 2015; Camagni et al., 2015). Dado que el tamaño no es una explicación suficiente y automática, se hace necesario considerar la dinámica urbana más general en la línea sugerida por Meijers et al. (2016). Según estos autores, existe un cierto excepcionalismo europeo consistente en un patrón urbano policéntrico y, sobre todo, en la importancia de las economías de red. Ello impide que se confirmen los modelos de la nueva geografía económica, que, al poner todo el énfasis en la aglomeración y el tamaño urbano como origen de externalidades positivas (Fujita et al., 1999; Glaeser, 2011), tienden a predecir el crecimiento de un menor número de ciudades más grandes. Para Dijkstra et al. (2013), los sistemas urbanos policéntricos estarían permitiendo en Europa un acceso mejorado a servicios en ciudades de menor tamaño, lo que incrementaría su atractivo frente a ciudades de mayor tamaño ya afectadas por deseconomías. La clave está en la conectividad externa, nacional e internacional, de las ciudades, que llega a ser un elemento más determinante que el propio tamaño para el comportamiento urbano, la atracción de funciones corporativas de alto rango y la competitividad global de las ciudades (McCann y Acs, 2011; Bel y Fageda, 2008; Ni y Kresl, 2010). La conectividad externa de las ciudades y su participación en redes complementarían a las externalidades ligadas a la aglomeración, de forma que estos beneficios podrían compartirse entre las ciudades conectadas en red. Las ciudades podrían así compensar su falta de tamaño o masa a través de un buen encuadramiento en redes urbanas (Camagni y Capello, 2015).

Para explicar el funcionamiento de estas redes urbanas, se ha acudido al concepto de "tamaño prestado» (borrowed size), formulado por Alonso (1973), que permitía explicar el buen comportamiento de pequeñas ciudades de entornos metropolitanos aprovechando las economías de aglomeración de la ciudad central. Pero, según Camagni et al. (2015) y Meijers y Burger (2015), estos procesos de "tomar prestado tamaño" no se producen solo por proximidad física a escala intrametropolitana, sino que también pueden darse con la participación en redes urbanas nacionales e internacionales a través de relaciones y flujos de carácter fundamentalmente horizontal y heterárquico $0^{1}$ entre ciudades complementarias o similares. Además, estas economías urbanas de red tienden a ser especialmente críticas en el campo empresarial y en la ciencia (Meijers y Burger, 2015).

La distribución espacial de la nueva economía del conocimiento en las principales áreas metropolitanas y en el conjunto de las regiones españolas, en ocasiones bajo el prisma de la economía creativa y de las clases creativas,

1. Utilizamos aquí el término heterárquico como característico de un sistema de organización que se distingue del jerárquico por favorecer la interrelación, la complementariedad y la cooperación entre sus miembros —en este caso las ciudades que componen un sistema urbano-, y en el que el poder y la capacidad de decisión están distribuidos de forma más equilibrada. 
ha ocupado una nutrida producción científica en los últimos años (Bergua et al., 2016; Boix y Lazzeretti, 2012; Caravaca et al., 2013; Escolano y Escalona, 2017; Méndez, 2013; Méndez et al., 2010; 2012; Pareja y Turmo, 2013; Sánchez, 2017; Sánchez et al., 2010; 2012; 2014). En este trabajo, y basándonos en el marco teórico expuesto, profundizaremos en el análisis del comportamiento que están teniendo las ciudades globales españoles, y en qué medida están liderando las transformaciones estructurales ligadas a la nueva economía global-informacional. En concreto, las diferentes trayectorias de cada ciudad permitirán valorar los procesos de dualización del mercado laboral que pudiera haber en curso, así como tratar de confirmar, en el caso español, las presuntas tendencias hacia una hipotética red urbana de carácter más heterárquico en la que las ciudades de segundo escalón mejoren su nivel funcional.

\section{Fuentes y metodología}

La mayoría de los análisis territoriales de la estructura productiva y ocupacional en España han utilizado como fuente de información el censo de población, registros de empleo de la Seguridad Social o, más recientemente, la muestra continua de vidas laborales. En este trabajo, en cambio, usaremos la Encuesta de población activa (EPA), principal fuente estadística sobre coyuntura del mercado laboral en España, con un detalle territorial superior al habitual, para aproximarnos al comportamiento de las cinco principales aglomeraciones metropolitanas españolas - Madrid, Barcelona, Valencia, Sevilla y Bilbao-, identificadas en las clasificaciones que elabora la red de investigación sobre globalización y ciudades mundiales (Globalization and World Cities-GaWC) dentro de la categoría de ciudades globales (GaWC, varios años). Estas cinco ciudades son también las únicas incluidas en las dos categorías superiores de la taxonomía de áreas metropolitanas españolas propuesta por Feria (2013) — regiones metropolitanas y áreas metropolitanas consolidadas - en las que los procesos urbanos han alcanzado una mayor madurez. Si bien las clasificaciones de otros autores (Rozenblat y Cicille, 2003; Lois y Piñeira, 2017; Escolano y Escalona, 2017) sugerirían la inclusión de otras ciudades (Málaga, Zaragoza, Granada o Palma de Mallorca), se ha preferido aquí optar por un criterio restrictivo para limitar el análisis a aquellas ciudades mayores que cumplen más claramente algún papel de control y coordinación global (Sassen, 1994).

Se utilizarán los microdatos de la EPA que recogen la información proporcionada por las 150.000-160.000 personas mayores de 16 años encuestadas cada trimestre y que permiten conocer para la población ocupada el sector de actividad económica, el grupo de ocupación del puesto de trabajo desempeñado, la provincia de residencia, así como, en el caso de que haya habido un cambio de residencia durante el año anterior, la provincia española o la zona del mundo de procedencia. La fuente permite conocer la estructura por actividades y ocupacional de los territorios, así como construir matrices origendestino de los flujos migratorios internos y cuantificar los flujos de inmigración procedentes del extranjero. Se trata de una información muy rica que permite 
un análisis en profundidad del nivel competencial de los diferentes territorios y los procesos de cambio en curso.

La EPA ofrece un detalle territorial de NUTS 3 (provincias). Tomando como elemento de contraste la delimitación metropolitana de las áreas urbanas funcionales (AUF) que contempla la iniciativa europea URBAN-AUDIT, su ajuste con las NUTS 3, aunque imperfecto, puede considerarse suficiente. En el caso de Madrid, la población residente en el AUF es ligeramente mayor $(+3 \%)$ que la de la provincia, fundamentalmente por su extensión hacia Toledo. En Barcelona y Bilbao, el AUF equivale al 90\%, lo que ofrece un ajuste satisfactorio, mientras que en Valencia y Sevilla las respectivas AUF delimitan territorios algo más pequeños: un $70 \%$ de la población provincial en Valencia y un $80 \%$ en el caso de Sevilla. En cualquier caso, consideramos que podemos utilizar la escala territorial de NUTS 3 como una aproximación suficiente al hecho metropolitano en estos cinco casos, máxime cuando esto nos permite explorar una fuente de información muy accesible y que facilita amplios análisis diacrónicos.

El detalle ocupacional con que contamos es de 10 grandes grupos de ocupación de la CNO-11, lo que permite identificar a los analistas simbólicos dentro del grupo 2 de Técnicos y profesionales científicos e intelectuales, y a muchos de los trabajadores de la subclase de servicios dentro del grupo 9 de Peones sin cualificar. Sin embargo, el detalle sectorial es de 10 agrupaciones de la CNAE09, insuficiente por sí solo para identificar actividades propias de la economía creativa o del conocimiento. Esto obliga a seguir una estrategia doble:

1) Con el fin de detectar a los analistas simbólicos, seleccionaremos solamente aquellos registros de individuos que pertenezcan al grupo de ocupación 2 de la CNO-2011 y que, simultáneamente, estén trabajando en los sectores de servicios más relacionados con la economía de la información, los servicios financieros, los servicios a la producción y la economía de la cultura (sectores 6, 7 y 9) de la clasificación de la EPA. De esa forma, la restricción ocupacional que establecemos permite poner el foco en los profesionales creativos y eliminar el empleo de menor cualificación y con funciones más banales.

2) Para contabilizar a los ocupados que componen la subclase de servicios urbanos, seleccionaremos todos los registros encuadrados en el grupo de ocupación 9 de la CNO-11 de Peones sin cualificar en los sectores construcción y servicios (agrupaciones de la EPA 4, 5, 6, 7, 8 y 9) y que recogen las actividades necesarias para el mantenimiento y la reproducción social y material de las áreas metropolitanas, pero que engloban puestos de trabajo de menor nivel competencial, a menudo mal pagados y con condiciones laborales precarias.

Esta doble aproximación sectorial/ocupacional presenta la ventaja de distinguir y clasificar separadamente a los ocupados que dentro del mismo sector económico de servicios a la producción desarrollan funciones de alta cualifi- 
cación (p. ej., analista de mercados) y de baja cualificación (p. ej., personal de limpieza). Aunque la metodología no es perfecta a la hora de separar y detectar en su totalidad a los integrantes de los dos grupos extremos de la escala de cualificaciones, permite explotar al máximo una fuente muy accesible y de actualización periódica y frecuente como es la EPA, y avanzar en el conocimiento de la evolución reciente de los procesos de cualificación/descualificación y dualización de los mercados de trabajo metropolitanos. Además, el enfoque adoptado excluye deliberadamente del análisis al personal altamente cualificado de los cuerpos superiores de la Administración pública y de los servicios asociados al estado de bienestar (salud, educación), porque sus dinámicas de localización obedecen en buena medida a lógicas no económicas de justicia social y espacial y contribuyen a una aparente homogeneización del territorio. Por esta razón, nuestra metodología detecta un numero de analistas simbólicos ligeramente inferior al de las aproximaciones únicamente ocupacionales, al dejar fuera al empleo público altamente cualificado en salud y educación. Consideramos, sin embargo, que este hecho es un activo de la metodología propuesta en la medida en que permite centrar el análisis en las dinámicas más propias del sistema productivo empresarial y en su capacidad para gestionar información. De la misma forma, esto nos ofrece la posibilidad de observar en toda su crudeza las disparidades interregionales.

Además, al poner el foco en las ocupaciones y no tanto en los sectores productivos, este enfoque de análisis de las competencias de los ocupados como reflejo del nivel funcional alcanzado por cada territorio pensamos que muestra una visión alternativa de gran interés. Creemos que la metodología cruzada aquí propuesta reporta ganancias analíticas frente a un enfoque puramente sectorial u ocupacional, al permitir trabajar con el segmento de empleados críticos para el manejo de información en la economía del conocimiento, al tiempo que la amplia serie temporal de los microdatos de la EPA permitirá una aproximación de carácter diacrónico, y observar los efectos que pudiera haber introducido la crisis económica en las dinámicas metropolitanas. El volumen de empleo que así observamos es claramente menor al de otras aproximaciones al tema en el caso español, pero de esta manera creemos que se gana en precisión analítica.

\section{Resultados y discusión}

\subsection{El nodo español de ciudades globales}

Los trabajos de la red de investigación GaWC-Globalization and World Cities permiten una primera aproximación a las dinámicas que están siguiendo las ciudades globales españolas. Uno de sus productos más conocidos es una clasificación de las ciudades globales, The world according to GaWC, basada en un índice de acceso y conectividad a servicios avanzados a la producción y servicios financieros, que es calculado a partir de la red de relaciones intraempresa a escala global. El valor de este índice de conectividad puede utilizarse como un 
Figura 1. Trayectorias de las ciudades globales españoles en la red mundial

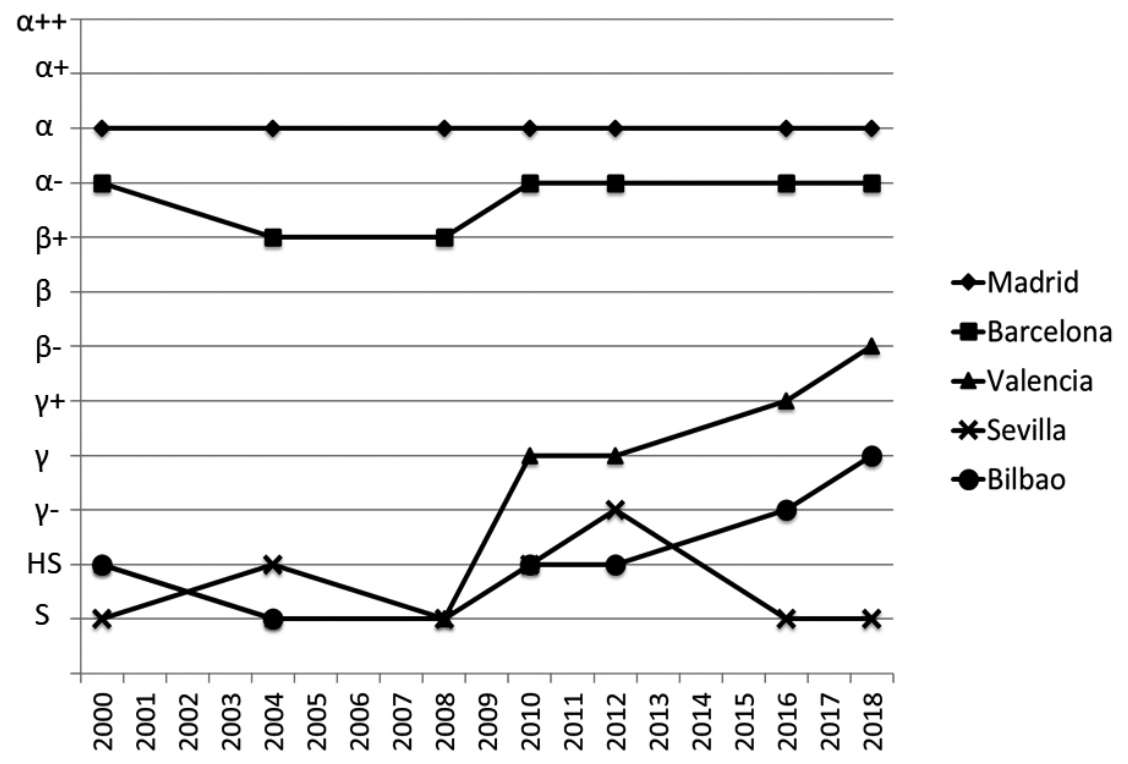

Fuente: GaWC (varios años), The world according the GaWC.

indicador del nivel funcional de cada la ciudad, del alcance territorial de su papel de control y coordinación, y de su acceso a información e innovaciones con efectos directos sobre su propia competitividad y la de su entorno regional (Taylor, 2001, 2012; Taylor et al., 2002; Derudder et al., 2010).

La trayectoria seguida por las ciudades globales españolas dentro de esta jerarquía mundial desde inicios del siglo Xxi (figura 1) muestra en primer lugar la constante y esperable preeminencia de Madrid, que se mantiene en la categoría a en todo momento, en el reconocimiento de su papel como nexo de integración de la economía española en la economía global y por su proyección hacia América Latina (Taylor et al., 2015). Sin embargo, sí se producen cambios muy significativos en el resto de las ciudades globales españolas de segundo escalón. Hasta 2008 su comportamiento podría calificarse de mediocre: Barcelona desciende una categoría, de $\alpha-$ a $\beta+$, mientras que Valencia, Sevilla y Bilbao se mantienen en las categorías más bajas (S-Suficiencia y HSAlta suficiencia). A partir de 2009 estas metrópolis experimentan una mejoría sensible en su nivel funcional, de forma especialmente clara en los casos de Barcelona, Valencia y Bilbao, con un incremento de su acceso y conectividad global a servicios avanzados. Estas trayectorias son coherentes con algunas de las conclusiones del programa ESPON 2013 relativas a la fuerte resiliencia y potencial de desarrollo de las ciudades metropolitanas del segundo escalo urbano (ESPON, 2012b), o al identificar a las regiones de Madrid, Cataluña, 
Tabla 1. Nivel medio competencial de la población ocupada. Escala de 1 a 4 según la CIUO88 de la OIT (índice 100 = valor de 2008)

\begin{tabular}{clrllll}
\hline Año & Madrid & Barcelona & Valencia & \multicolumn{1}{c}{ Sevilla } & \multicolumn{1}{c}{ Bilbao } & España \\
\hline 2005 & $2,44(97)$ & $2,39(101)$ & $2,30(99)$ & $2,33(98)$ & $2,48(99)$ & $2,32(99)$ \\
2008 & $2,53(100)$ & $2,38(100)$ & $2,32(100)$ & $2,39(100)$ & $2,50(100)$ & $2,35(100)$ \\
2013 & $2,66(105)$ & $2,46(103)$ & $2,35(101)$ & $2,42(102)$ & $2,53(101)$ & $2,40(102)$ \\
2019 & $2,61(103)$ & $2,52(106)$ & $2,38(103)$ & $2,45(103)$ & $2,53(101)$ & $2,41(102)$ \\
\hline
\end{tabular}

Fuente: microdatos de la Encuesta de población activa (INE).

Comunidad Valenciana y País Vasco-Navarra-La Rioja como las que, en el caso español, presentan mejores perspectivas en un contexto de globalización (ESPON, 2012a). El análisis propuesto para estas cinco ciudades globales permitirá comprobar hasta qué punto se están produciendo transformaciones en su estructura productiva y ocupacional coherentes con este pretendido proceso de reforzamiento del segundo escalón metropolitano.

El nivel competencial medio de la población ocupada (tabla 1) mejora de forma generalizada. En el período anterior a la crisis, Madrid destaca por la rapidez con la que gana nivel competencial, muy por encima del resto, con una tendencia a largo plazo de fortalecimiento de la primacía que ostenta la ciudad cabecera. No obstante, la crisis iniciada en 2008 parece haber contribuido a una mitigación de esta tendencia. Una ciudad del segundo escalón, Barcelona, es la más dinámica, mientras que el resto equipara su crecimiento al de Madrid, especialmente a partir de 2013. Esta mejora general del nivel competencial de las ciudades del segundo escalón metropolitano a partir de la crisis del 2008, que continua y se refuerza con la recuperación iniciada en 2013, es coherente con lo que sería de esperar a partir de los análisis globales de la red GaWC y de lo propuesto desde el programa ESPON 2013.

\subsection{La salida de la crisis y el papel del segundo escalón urbano/metropolitano}

La pauta de localización de los analistas simbólicos — de los trabajadores del conocimiento- es de una extrema concentración en los escalones superiores de la jerarquía urbana (tabla 2). Tomando como referencia 2008, momento final del prolongado ciclo de crecimiento anterior, Madrid reúne por sí sola a más de $1 / 3$ del total $(34,2 \%)$ y Barcelona casi $1 / 6$ (14,9\%). Conjuntamente, las dos principales áreas metropolitanas del país suman casi la mitad de estas ocupaciones en España $(49,1 \%)$, mientras que las tres siguientes en tamaño —Valencia, Sevilla y Bilbao- reúnen un 13,2\% adicional. De esta, forma, cerca de 2/3 del capital humano más cualificado empleado en los sectores intensivos en el uso de conocimiento — servicios avanzados, sector financiero y sector cultural - se concentra en las cinco principales aglomeraciones urbanas. Este nivel de concentración es superior al que detecta Méndez (2013) para el conjunto de trabajadores en el continuo servindustrial de la economía del conocimiento, pero muy similar al que Sánchez y Arellano (2012) 
Analistas simbólicos, jerarquía urbana y atracción de talento en las

Tabla 2. Distribución territorial de los analistas simbólicos. Grupo de ocupación 2 en sectores 6,7 y 9 de la EPA. \% sobre el total de España e índices de concentración territorial (50 provincias)

\begin{tabular}{ccccccccc}
\hline Año & Madrid & Barcelona & Valencia & Sevilla & Bilbao & 5 ciudades globales & HHI & Gini \\
\hline 2005 & $32,7 \%$ & $16,3 \%$ & $5,5 \%$ & $3,6 \%$ & $3,7 \%$ & $61,9 \%$ & 1.443 & 0,742 \\
2008 & $34,2 \%$ & $14,9 \%$ & $5,3 \%$ & $3,9 \%$ & $4,0 \%$ & $62,3 \%$ & 1.501 & 0,737 \\
2013 & $34,3 \%$ & $15,9 \%$ & $3,9 \%$ & $3,4 \%$ & $3,4 \%$ & $60,7 \%$ & 1.518 & 0,743 \\
2019 & $32,3 \%$ & $16,6 \%$ & $4,3 \%$ & $3,9 \%$ & $2,5 \%$ & $59,6 \%$ & 1.418 & 0,739 \\
\hline
\end{tabular}

Fuente: microdatos de la Encuesta de población activa (INE).

encuentran para los trabajadores más cualificados de los sectores intensivos en conocimiento.

Esta disposición territorial muestra una enorme inercia a lo largo del tiempo. No obstante, es posible observar algunas ligeras variaciones que afectan tanto a su distribución general en toda España como al peso específico que tienen las cinco principales áreas metropolitanas. Así, la concentración territorial de los analistas simbólicos (tabla 2 y figura 2) se incrementó notablemente en la fase de recesión (2008-2013), mientras que disminuye en los años de bonanza económica (2005-2008 y 2013-2019). La evolución del índice de Gini a lo largo de todo el período de estudio se ajusta a un comportamiento

Figura 2. Concentración territorial de los analistas simbólicos (2005-19). Grupo de ocupación 2 en sectores 6,7 y 9 de la EPA. Índice de Gini y ajuste de una función polinómica (50 provincias)

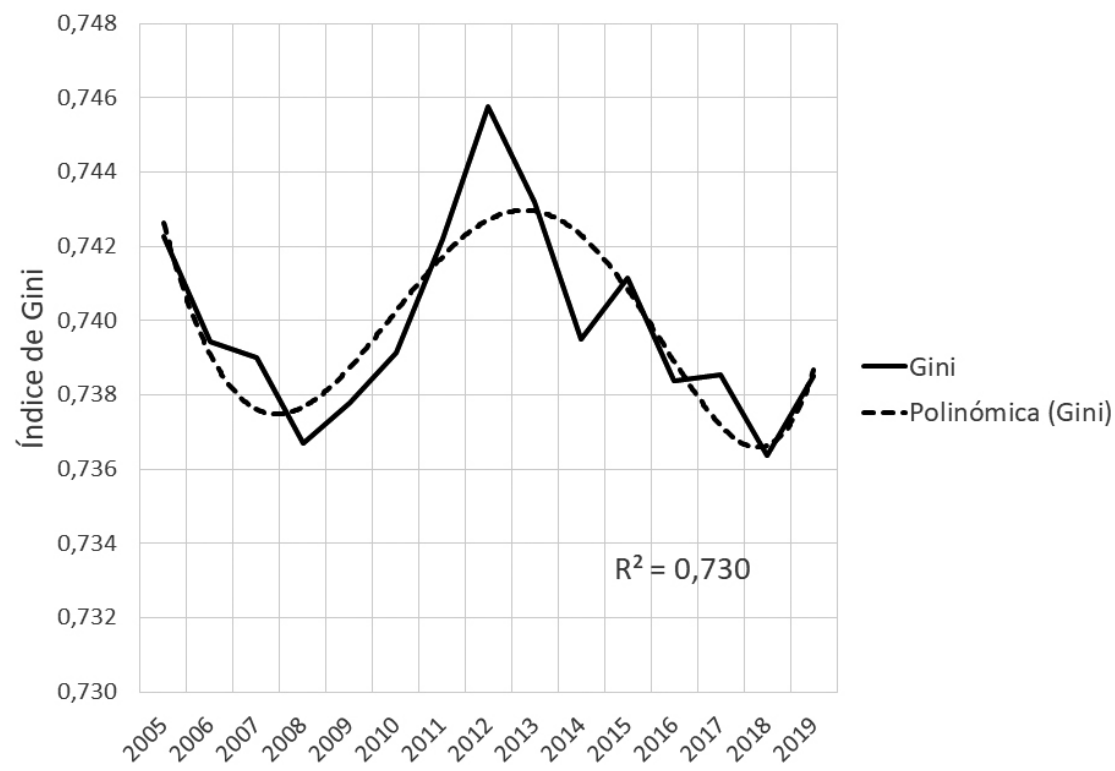

Fuente: microdatos de la Encuesta de población activa (INE). 
cíclico $\left(\mathrm{R}^{2}\right.$ de 0,730 a una función polinómica): crisis y períodos de expansión tienen efectos asimétricos en el territorio.

En los primeros años de la crisis iniciada en 2008, las funciones de servicios avanzados y las actividades intensivas en conocimiento sobrevivieron mejor y continuaron mejor su expansión en Madrid y Barcelona, mientras que en el resto de las ciudades de segundo escalón la dinámica fue más mediocre. Probablemente, esta inicial evolución diferencial se explique porque los servicios de mayor complejidad y nivel jerárquico se han comportado mejor aprovechando las importantes economías de aglomeración de Madrid y Barcelona, lo que ha dado lugar a un proceso de repliegue hacia ellas, al tiempo que actividades financieras y de servicios avanzados muy ligados a la economía de la burbuja inmobiliaria dominante antes de 2008 sufrían un castigo especialmente alto en las ciudades del segundo escalón metropolitano. Esto puede haber sido especialmente cierto para los servicios financieros u orientados a empresas que Escolano y Escalona (2017) identifican como los que presentan superiores tendencias a la difusión territorial vía creación de filiales y que más han sufrido un repliegue con la crisis. Esto es coherente no solo con la evolución del índice de Gini durante la crisis, sino también con los valores que toma el HerfindahlHirschman Index (HHI), índice de concentración que se ve mucho más afectado por lo que sucede en la cabecera del sistema, y que alcanza su máximo en 2013, al final del período de recesión, cuando Madrid reúne el 34,2\% del empleo español de analistas simbólicos (tabla 2).

A partir de 2013, las funciones urbanas de obtención, generación y difusión de información y conocimiento actúan como motor del crecimiento al constituirse en un sector básico de las economías urbanas, y también como condición necesaria para el desarrollo del resto de actividades, muy especialmente si están trabajando para mercados globales e internacionales. Como estrategia de salida a la crisis de 2008, la economía española se proyecta de forma muy intensa hacia el exterior, de forma que el peso de las exportaciones de bienes y servicios sobre el PIB pasa entre 2008 y 2019 del 23,1\% al 35,0\% (Banco de España, 2020). Este modelo de desarrollo hacia fuera, de creciente integración en los mercados globales, ha ido acompañado de un crecimiento continuo y sin precedentes del estrato ocupacional de analistas simbólicos, muy visible en las economías regionales más dinámicas y volcadas al exterior. Así, en la fase de crecimiento que se inicia en 2013, aunque su concentración territorial sigue siendo muy elevada, se puede observar una cierta tendencia descentralizadora que se advierte tanto en el descenso del índice de Gini como especialmente del HHI, que registra su mínimo en 2019, cuando refleja la pérdida relativa de peso de Madrid, que baja hasta el 32,3\% del total español.

Al mismo tiempo, durante esta coyuntura expansiva y de creciente conexión exterior, y como expresión territorial de esta nueva dinámica, destacan tres ciudades del segundo escalón (Barcelona, Valencia y Sevilla), que incrementan conjuntamente su participación en el total español 1,6 puntos (del 23,4\% al $24,8 \%$ ). Esta dinámica positiva experimentada por este segundo escalón metropolitano es compartida por otras aglomeraciones urbanas importantes de 
Analistas simbólicos, jerarquía urbana y atracción de talento en las

Tabla 3. Participación de los analistas simbólicos en la estructura productiva. Grupo de ocupación 2 en sectores 6,7 y 9 de la EPA. \% sobre ocupados en cada territorio (índice $100=2008)$ <Cociente de localización>

\begin{tabular}{rrrrrrr}
\hline Año & Madrid & Barcelona & Valencia & Sevilla & Bilbao & 5 ciudades globales \\
\hline 2005 & $6,7 \%(80)$ & $3,9 \%(89)$ & $3,1 \%(89)$ & $3,0 \%(75)$ & $4,4 \%(72)$ & $4,8 \%(81)$ \\
& $<2,16>$ & $<1,26>$ & $<1,00>$ & $<0,97>$ & $<1,42>$ & $<1,54>$ \\
2008 & $8,4 \%(100)$ & $4,4 \%(100)$ & $3,5 \%(100)$ & $4,0 \%(100)$ & $6,1 \%(100)$ & $5,9 \%(100)$ \\
& $<2,21>$ & $<1,16>$ & $<0,92>$ & $<1,05>$ & $<1,61>$ & $<1,55>$ \\
2013 & $11,1 \%(132)$ & $6,4 \%(145)$ & $3,7 \%(106)$ & $4,7 \%(118)$ & $6,5 \%(107)$ & $7,8 \%(132)$ \\
& $<2,18>$ & $<1,25>$ & $<0,73>$ & $<0,92>$ & $<1,27>$ & $<1,53>$ \\
2019 & $11,4 \%(136)$ & $7,1 \%(161)$ & $4,4 \%(126)$ & $5,7 \%(143)$ & $5,7 \%(93)$ & $8,2 \%(139)$ \\
& $<2,08>$ & $<1,29>$ & $<0,80>$ & $<1,04>$ & $<1,04>$ & $<1,49>$ \\
\hline
\end{tabular}

Fuente: microdatos de la Encuesta de población activa (INE).

índole regional. Málaga, Zaragoza, La Coruña, Valladolid y Murcia incrementan conjuntamente su participación desde el 9,4\% al 10,7\%. Sin embargo, este proceso de difusión territorial solo se traduce en la potenciación del segundo escalón metropolitano y de estas capitales regionales de mayor tamaño, sin que afecte al resto del territorio, lo que, por otra parte, es coherente con la fuerte vocación urbana y metropolitana de este este tipo de funciones (Escolano y Escalona, 2017).

Los cocientes de localización de los analistas simbólicos, calculados en comparación con su presencia en la estructura ocupacional española (tabla 3), son plenamente coherentes con la evolución descrita, pues son crecientes entre 2013 y 2019 en Barcelona, Valencia y Sevilla, y muestran la diferente intensidad con que cada ciudad global está avanzando en el proceso de transformación y adaptación a una economía global en la que los analistas simbólicos cumplen un papel central. La crisis nunca detuvo la evolución ascendente del empleo de este grupo de profesionales, que ha seguido creciendo en volumen absoluto en todo momento y en todos los casos de estudio, incluso en la coyuntura de recesión, lo que ha determinado una transformación acelerada de las estructuras productivas y de ocupación (tabla 4). Estas tendencias son previas a la crisis de 2008 y, con la sola excepción de Bilbao, continúan y hasta se profundizan con posterioridad. Al final del periodo observado, estos profesionales del conocimiento alcanzan una presencia elevadísima en Madrid (11,4\% de todo el empleo metropolitano en 2019) y notable en Barcelona (7,1\%), en Sevilla y Bilbao (5,7\% en ambos casos). Solo Valencia muestra una posición algo más atrasada, con una presencia relativa llamativamente baja de este tipo de profesionales, un síntoma claro de la persistencia de marcados déficits funcionales, al menos con relación a lo que sería de esperar atendiendo a su tamaño demográfico y económico.

Bilbao es quizás la mayor excepción al buen comportamiento relativo que por lo general están mostrando las ciudades globales del segundo escalón metropolitano a partir de 2013. Aunque su nivel medio competencial es 
relativamente alto y creciente, el grupo ocupacional de analistas simbólicos integrados en el sector de servicios avanzados presenta una evolución bastante peor que en el resto de las ciudades analizadas, e incluso se llega a observar una reducción en su número absoluto entre 2013 y 2019, algo realmente inusitado. Una explicación plausible a esta excepción podría venir de la mano de la propia estructura productiva vasca, en la que la industria manufacturera mantiene un gran peso. En este caso, los procesos de cualificación ocupacional y modernización productiva podrán ser diferentes a los de las otras ciudades globales, sin que el sector de servicios avanzados tenga el mismo papel.

\subsection{Procesos de dualización ocupacional}

Es de destacar que la primacía de Madrid se mantiene, de manera que la presencia de estos profesionales/funciones ligada a la economía del conocimiento sigue creciendo aquí a un ritmo notable en términos absolutos (tabla 4), sobre todo si atendemos al gran volumen que este tipo de ocupaciones han alcanzado en la ciudad cabecera del sistema urbano español. Su volumen, con 350.000 efectivos en 2019 , se ha multiplicado por 1,8 desde 2005 y por 1,3 desde 2008. Pero, junto con esta indiscutible primacía de Madrid, Barcelona muestra una dinámica muy intensa (180.000 efectivos en 2019, con factores de crecimiento de $\times 1,9$ desde 2005 y $\times 1.6$ desde 2008). También Valencia y Sevilla se unen a esta dinámica expansiva de forma clara a partir de 2013.

Estas dinámicas de expansión de las ocupaciones de mayor cualificación explican en buena parte el incremento del nivel competencial que vimos que están experimentando las ciudades globales (tabla 1). Pero, además, este proceso se ve apoyado también en la caída muy ostensible de los ocupados en la subclase de servicios de menor cualificación competencial. En contra de lo que en ocasiones se postula, estos grupos de ocupados están perdiendo peso de forma generalizada en la estructura ocupacional de las ciudades globales españolas: entre 2005 y 2019 han perdido cerca de 140.000 efectivos, y su peso en la estructura ocupacional ha caído desde el 11,8\% al 9,7\% (tabla 4). Su fuerte descenso en los años de recesión se ha recuperado solo muy parcialmente durante la recuperación posterior. En 2019 solo se había recuperado uno de cada cinco empleos perdidos en este estrato ocupacional durante la crisis. De esta manera, no encontramos sustento empírico claro para la hipótesis de la dualización de las estructuras ocupaciones en las metrópolis globales. En el conjunto de las cinco ciudades globales españolas, incluso en las fases expansivas, los analistas simbólicos crecen mucho más deprisa que la subclase de servicios, y esta última lo hace más despacio que el conjunto de la ocupación. Solo Madrid en los años más recientes (2013-19) se escapa a esta norma general y podría ser un ejemplo de dualización ocupacional: por cada puesto de trabajo como analista simbólico se ha creado otro en la subclase de servicios, y ambos extremos de cualificación están perdiendo peso relativo simultáneamente (tabla 4). 
Tabla 4. Volumen absoluto de analistas simbólicos y de trabajadores en la subclase de servicios. \% en la estructura ocupacional metropolitana. Grupo de ocupación 2 en sectores 6,7 y 9 de la EPA y grupo de ocupación 9 en los sectores 4, 5, 6, 7, 8 y 9 de la EPA

\begin{tabular}{|c|c|c|c|c|c|c|c|c|c|c|c|c|}
\hline \multirow[b]{2}{*}{ Año } & \multicolumn{6}{|c|}{ Analistas simbólicos } & \multicolumn{6}{|c|}{ Trabajadores en la subclase de servicios urbanos } \\
\hline & $\begin{array}{l}\text { 은 } \\
\text { 疍 }\end{array}$ & 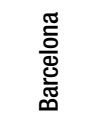 & $\frac{\frac{\pi}{0}}{\frac{\frac{\pi}{0}}{\pi}}$ & 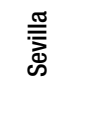 & 忍 & 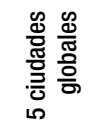 & $\begin{array}{l}\text { 은 } \\
\text { 吾 }\end{array}$ & 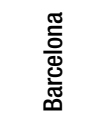 & $\frac{\frac{\pi}{0}}{\frac{0}{\pi}}$ & 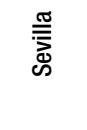 & 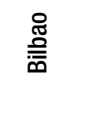 & 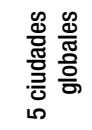 \\
\hline \multirow[t]{2}{*}{2005} & 195.730 & 97.705 & 33.144 & 21.528 & 22.002 & 370.108 & 385.638 & 242.371 & 140.663 & 84.94 & 56.712 & 910.328 \\
\hline & $6,7 \%$ & $3,9 \%$ & $3,1 \%$ & $3,0 \%$ & $4,4 \%$ & $4,8 \%$ & $13,2 \%$ & $9,6 \%$ & $13,0 \%$ & $11,9 \%$ & $11,2 \%$ & $11,8 \%$ \\
\hline \multirow[t]{2}{*}{2008} & 263.947 & 115.372 & 41.021 & 30.080 & 31.092 & 481.512 & 384.197 & 282.445 & 142.898 & 95.587 & 56.488 & 961.615 \\
\hline & $8,4 \%$ & $4,4 \%$ & $3,5 \%$ & $4,0 \%$ & $6,0 \%$ & $5,9 \%$ & $12,3 \%$ & $10,6 \%$ & $12,3 \%$ & $12,6 \%$ & $10,9 \%$ & $11,7 \%$ \\
\hline \multirow[t]{2}{*}{2013} & 301.624 & 139.967 & 34.471 & 28.783 & 29.370 & 535.576 & 260.679 & 206.320 & 118.123 & 61.663 & 44.676 & 691.461 \\
\hline & $11,1 \%$ & $6,4 \%$ & $3,7 \%$ & $4,7 \%$ & $6,5 \%$ & $7,8 \%$ & $9,6 \%$ & $9,5 \%$ & $12,8 \%$ & $10,0 \%$ & $9,8 \%$ & $10,0 \%$ \\
\hline \multirow[t]{2}{*}{2019} & 352.201 & 181.192 & 47.402 & 42.066 & 27.445 & 650.307 & 319.551 & 220.806 & 116.892 & 71.540 & 41.479 & 770.267 \\
\hline & $11,4 \%$ & $7,1 \%$ & $4,4 \%$ & $5,7 \%$ & $5,7 \%$ & $8,2 \%$ & $10,3 \%$ & $8,7 \%$ & $10,9 \%$ & $9,7 \%$ & $8,6 \%$ & $9,7 \%$ \\
\hline
\end{tabular}

Fuente: microdatos de la Encuesta de población activa (INE).

\subsection{El papel de las migraciones}

Las migraciones, tanto internas como internacionales, pueden afectar el patrón de distribución territorial de los analistas simbólicos. Usualmente se atribuye a este tipo de profesionales una cierta libertad locacional que los lleva a elegir su lugar de residencia y trabajo en función de consideraciones no solo laborales, sino también de ambiente social y económico (tolerancia, calidad de vida, creatividad, cultura local), lo que generaría una fuerte competencia interurbana por el talento (Florida, 2007; Musterd et al., 2016). Sin embargo, de acuerdo con la evidencia empírica que hemos recogido, este factor podría estar sobrestimado en el caso español. Según la información proveniente de los microdatos de la EPA, en el conjunto del período 2005-2019 solo un 0,61\% de estos profesionales del conocimiento cambiaron anualmente de provincia de residencia dentro de España. Existe también un flujo de llegada desde el extranjero que moviliza un volumen de población similar, y que en este mismo periodo supuso un aporte del $0,50 \%$ anual. Tal y como sería de esperar, en los flujos que se producen en el interior de España, las cinco ciudades globales tienen una participación algo menor $(43,4 \%)$ que en los que proceden del extranjero $(63,7 \%)$ : las menores incertidumbres y un mercado laboral de mayor tamaño y más diverso permiten explicar esta preferencia por las principales ciudades de los migrantes internacionales.

El flujo total de llegada de analistas simbólicos a cada ciudad global puede utilizarse como indicador de su atractivo para este tipo de profesionales, tanto por las oportunidades que ofrece para el desarrollo de su carrera profesional como por las características de su ambiente socioeconómico (figura 3). Se observan importantes variaciones interterritoriales relacionadas con los efectos de la crisis: Barcelona y Valencia, que fueron los territorios donde más 
Figura 3. Atracción de talento. Llegadas de analistas simbólicos en servicios avanzados. Tasa anual de inmigración (\%)

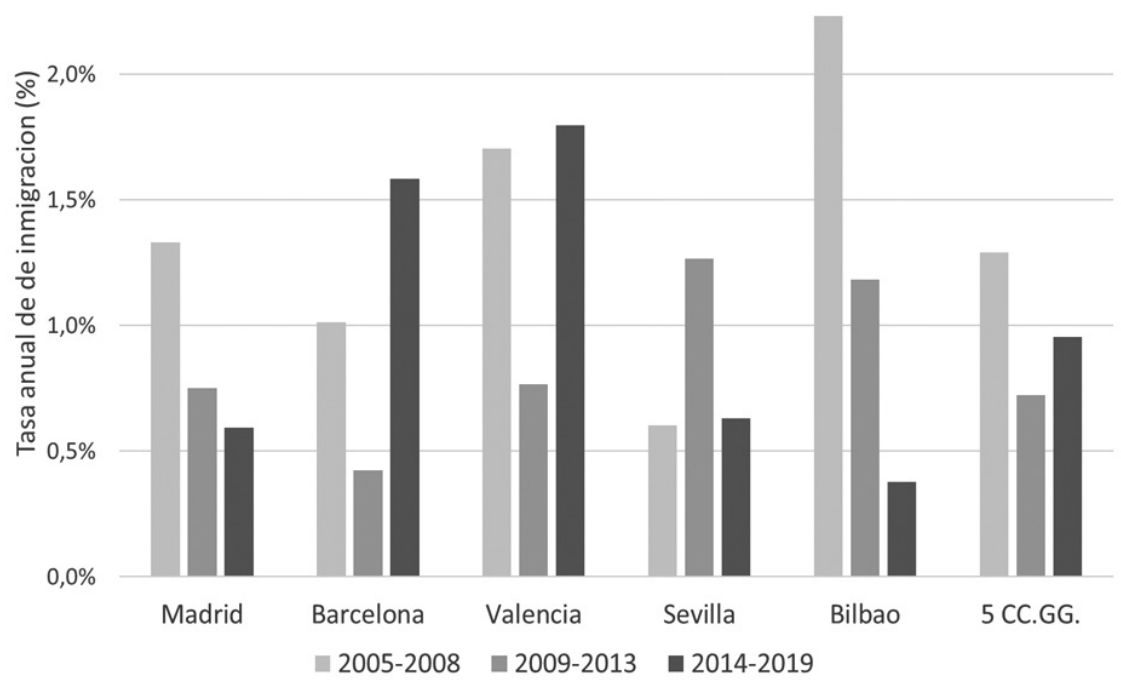

Fuente: microdatos de la Encuesta de población activa (INE).

se dejó sentir la crisis, con una fuerte caída de la llegada de profesionales, han tomado posiciones de liderazgo en la actualidad. De nuevo, atendiendo a lo ocurrido en los últimos años, no parece que los flujos migratorios estén ayudando a incrementar la primacía del sistema: Madrid muestra una tasa de inmigración claramente por debajo de la de Barcelona o Valencia. Bien al contrario, la movilidad territorial de los analistas simbólicos estaría contribuyendo, si bien de forma limitada por su escaso volumen, a fortalecer la posición de algunas de las ciudades que componen el segundo escalón metropolitano, tal y como también sugieren Sánchez et al. (2018). Por otro lado, el eje de desarrollo mediterráneo, especialmente afectado por la crisis derivada del estallido de la burbuja inmobiliaria y la crisis financiera, parecería por tanto haber vuelto al primer plano como uno de los motores principales del crecimiento español a partir de 2013. Así, en los casos de Valencia y Barcelona, su fuerte atractivo migratorio para este personal altamente cualificado se ve además acompañado por una contribución especialmente elevada de inmigración internacional procedente no solo de los países ricos de la Europa de los 15, sino también de países emergentes y particularmente de América Latina. Sin embargo, este reciente atractivo migratorio no ha sido capaz todavía de revertir algunas situaciones de fuerte déficit funcional y de pobreza en el desarrollo de la economía del conocimiento, como pone de manifiesto el caso de Valencia. 


\section{Conclusiones}

En el marco del proceso de modernización productiva y cualificación funcional que acompaña a la cada vez más profunda integración global de la economía española, el nodo español de ciudades globales está transformándose. La acusada primacía de Madrid está suavizándose ligera y paulatinamente en favor de estructuras más equilibradas como consecuencia del buen comportamiento relativo experimentado por Barcelona desde el mismo comienzo de la crisis, que se extiende claramente a Valencia y Sevilla en la salida de la recesión a partir de 2013. Las tendencias actuales, en una coyuntura de fuerte crecimiento y transformación estructural, parecen ir hacia una red de relaciones intermetropolitanas en la que la jerarquía y la fuerte primacía de Madrid se va suavizando. Según esto, estaríamos asistiendo a un proceso de difusión de las funciones más complejas de gestión del conocimiento no solo hacia el segundo escalón metropolitano (Barcelona, Valencia, Sevilla), sino también hacia las principales capitales regionales. La hipótesis resultante que aquí se propone sería la aparición de un sistema intermetropolitano más heterárquico, en el que las relaciones de complementariedad y colaboración irían ganando espacio frente a las de hegemonía y concentración del poder de decisión. En este sentido, una posible línea de investigación futura para confirmar esta hipótesis podría dirigirse a medir las economías externas derivadas de la participación en redes de que disfruta cada área metropolitana y ponerlas en relación con su mayor o menor dinamismo económico. En suma, se trataría de explorar la aplicación del concepto de "tamaño prestado» enunciado por Alonso a las relaciones intermetropolitanas.

La reciente explosión del empleo de analistas simbólicos en las ciudades de segundo escalón metropolitano (Barcelona, Sevilla y Valencia) y otras capitales regionales (Zaragoza, Málaga, Murcia, Valladolid, La Coruña) no puede dejar de ponerse en relación con el renovado modelo de crecimiento orientado hacia el exterior. De hecho, podríamos incluso considerar que ha sido una condición necesaria para ello, dado que sus exigencias en términos de capacidad para innovar y gestionar información son claramente superiores. Este proceso ha sido fundamental para el incremento reciente del nivel medio competencial de la población ocupada, que está siendo incluso más rápido en las ciudades globales que en el resto del territorio. De esta forma, se está facilitando la conexión global de las economías regionales. Desde esta perspectiva, las principales ciudades del sistema urbano español, y no solo Madrid, estarían mostrando una notable capacidad de reacción y resiliencia, y estarían lejos de ser meros actores pasivos del proceso de globalización.

Por otro lado, encontramos escasa evidencia, limitada al caso de Madrid, de que esté teniendo lugar un proceso de dualización de la estructura ocupacional por cualificaciones. Las ciudades del segundo escalón metropolitano están reduciendo con claridad la presencia de puestos de trabajo de muy baja cualificación (peones sin calificar) en sus estructuras ocupacionales, incluso en 
los casos de Valencia y Sevilla, donde se parte de niveles relativamente altos, lo que contrasta con la evolución diametralmente opuesta que exhibe Madrid. Aparentemente, el modelo de desarrollo de las ciudades de segundo escalón es más sostenible socialmente, aunque quizás lo que estamos observando es simplemente una convergencia del conjunto de ciudades globales hacia estructuras ocupacionales más similares y homogéneas. En cualquier caso, la aparición de tendencias a la precarización incluso en el segmento de trabajadores del conocimiento (Méndez, 2013; Sánchez et al., 2014), fruto de la implantación de nuevos sistemas de relaciones laborales crecientemente flexibles, hace todavía más complejo interpretarlas.

La tasa de inmigración total (llegadas nacionales+internacionales) puede utilizarse como un indicador del atractivo que cada territorio tiene para el talento. Así, en los últimos años las dos grandes áreas metropolitanas del litoral mediterráneo, Barcelona y Valencia, que fueron muy afectadas por la crisis, han pasado a liderar el atractivo para el talento extranjero, tanto a escala nacional como internacional, y han superado a Madrid y actuado como un factor de primer nivel para reducir su primacía. El eje mediterráneo de desarrollo estaría así dando de nuevo muestras de su recuperación una vez superada la crisis, al integrar en su estructura ocupacional a estos inmigrantes altamente cualificados, una parte importante de los cuales procede del extranjero. En futuras investigaciones sería interesante explorar qué papel están ejerciendo en cada territorio los factores de tipo soft (calidad de vida, entorno urbano, servicios urbanos) y hard (mercado laboral, oportunidades económicas), que pueden explicar estas diferencias de atractivo (Musterd y Murie, 2010), sin olvidar la conectividad externa (física y virtual) de cada lugar, que permite disfrutar de economías urbanas de red.

De nuestros resultados también puede derivarse alguna conclusión desde el punto de vista de las políticas públicas. Pensamos que el presente trabajo da argumentos en favor de estrategias de intervención y gasto público que atiendan a las necesidades de las ciudades del segundo escalón urbano y metropolitano, particularmente en materia de conectividad externa. Su eficiencia e impacto serían mayores que los de una estrategia centrada en Madrid como ciudad cabecera del sistema, al permitir el reforzamiento de unas potencialidades presentes en las ciudades de segundo escalón metropolitano y que ya se están manifestando de forma espontánea. Se trataría de poner el foco sobre la potenciación de las economías externas de red urbana, dentro de una concepción en la que el propio tamaño ya no es la única fuente de economía de aglomeración, y en la que la conexión con otras ciudades globales no debe ser vista necesariamente como un elemento succionador de riqueza, actividad y funciones superiores, sino como una oportunidad para acceder a recursos, información y oportunidades. Las nuevas tecnologías de la información y la comunicación, complementadas con una mejorada accesibilidad física, abren esta ventana de oportunidad, que, lejos de concepciones de «suma cero», pude permitir dinámicas de desarrollo compartido. 
Finalmente, desde un punto de vista metodológico, consideramos que queda probado que la EPA es una fuente de información válida para el estudio territorial de la economía del conocimiento en España. El trabajo con microdatos ofrece la posibilidad de realizar estudios cruzados (ocupación/actividad) que permiten acotar el objeto de análisis, mientras que las limitaciones derivadas de su detalle territorial se ven ampliamente compensadas por la posibilidad de realizar estudios diacrónicos amplios.

\section{Referencias bibliográficas}

Albertos, Juan Miguel (2019). «Trayectorias económicas de las regiones españolas en un escenario post-crisis: efectos de vecindad, especialización y aglomeración». Revista de Estudios Andaluces, 38, 8-27. $<$ https://doi.org/10.12795/rea.2019.i38.01>

Albertos, Juan Miguel y SÁnchez Hernández, José Luis (2017). «Caracterización y especialización industrial y del terciario avanzado en las regiones españolas». En: Farinós, Joaquín y Olcina, Jorge (eds.). Geografia regional de España. Espacios y comunidades. Valencia: Tirant Humanidades, 291-370.

Alonso, William (1973). «Urban zero population growth». Daedalus, 109, 191-206. Banco de España (2020). Boletín Estadístico, 2/2020. Madrid: Banco de España.

Barrios, Candelari; Flores, Esther y Martínez, María Ángeles (2017). «Patrones de convergencia en las regiones españolas: una aplicación de la metodología de Philpps-Sul». Revista de Estudios Regionales, 109, 165-190.

BeL, Germà y FAGEDA, Xavier (2008). «Getting there fast: Globalization, intercontinental flights and location of headquarters». Journal of Economic Geography, 8, 471-495. <https://doi.org/10.1093/jeg/lbn017>

Bergua, José Ángel; Pac, David: Báez, Juan Miguel y Serrano, Cecilia (2016). «La clase creativa: una aproximación a la realidad española». Revista Internacional de Sociología, 74, 1-19. <https://doi.org/10.3989/ris.2016.74.2.032>

Borx, Rafael y LazzeretTi, Luciana (2012). «Las industrias creativas en España: una panorámica». Investigaciones Regionales, 22, 181-206.

CAMAGNI, Roberto y CaPello, Roberta (2015). «Second Rank City Dynamics: Theoretical Interpretations Behind Their Growth Potentials». European Planning Studies, 23, 1.041-1.053. <https://doi.org/10.1080/09654313.2014.904994>

Camagni, Roberto; Capello, Roberta y Caragliu, Andrea (2015). «The Rise of Second-Rank Cities: What Role for Agglomeration Economies?». European Planning Studies, 23, 1.069-1.089. $<$ https://doi.org/10.1080/09654313.2014.904999>

Caravaca, Inmaculada; González, Gema; Fernández, Víctor y García, Antonio (2013). «Economía creativa en la aglomeración metropolitana de Sevilla: agentes, redes locales de colaboración y principales actuaciones». Boletin de la Asociación de Geógrafos Españoles, 63, 81-103. <https://doi.org/10.21138/bage.1607>

CAstells, Manuel (1996). The Information Age: Economy, Society and Culture, Cambridge Mass.: Blackwell. [Ed. cast. (1997). La era de la información: economía, sociedad y cultura. Madrid: Alianza Editorial]. 

ciudades mundiales españolas (2005-2019): un análisis a partir de la EPA

Daniels, Peter W. y Bryson, John, R. (2002). «Manufacturing Services and Servicing Manufacturing: Knowledge-based Cities and Changing Forms of Production». Urban Studies, 39, 977-991. <https://doi.org/10.1080/00420980220128408>

Derudder, Ben; TAylor, Peter. J. y Ni, Pengfei (2010). «Pathways of Change: Shifting Connectivities in the World City Network, 2000-08». Urban Studies, 47, 1.861-1.877. <https://doi.org/10.1177/0042098010372682>

Dijnstra, Lewis; Garcilazo, Enrique y McCann, Philip (2013). «The Economic Performance of European Cities and City Regions: Myths and Realities». European Planning Studies, 21, 334-354. <https://doi.org/10.1080/09654313.2012.716245>

Escolano, Severino y Escalona, Ana (2017). Especialización, concentración y aglomeración espacial. Documents d'Anàlisi Geogràfica, 63 (1), 55-80. <https://doi.org/10.5565/rev/dag.328>

ESPON (2012a). TIGER - Territorial Impact of Globalization for Europe and its Regions, Final Report. Luxemburgo: ESPON Coordination Unit.

- (2012b). SGPTD - Secondary Growth Poles and Territorial Development in Europe: Performance, Policies and Prospects. Luxemburgo: ESPON Coordination Unit.

Feria, José María (2013). «Hacia una taxonomía de las áreas metropolitana españolas». Boletín de la Asociación de Geógrafos Españoles, 63, 499-506. <https://doi.org/10.21138/bage.1618>

Florida, Richard (2002). The Rise of the Creative Class. And How It's Transforming Work, Leisure, Community and Everyday Life. Nueva York: Basic Books. $<$ https://doi.org/10.25071/1705-1436.180>

- (2007). The Flight of the Creative Class. The New Global Competition for Talent. Nueva York: Basic Books.

Friedmann, John (1986). «The World City Hypothesis». Development and Change, $17,69-83$.

Fujita, Masahisa; Krugman, Paul y Venables, Anthony (1999). The Spatial Economy. Cambridge Mass.: MIT Press.

GAWC (varios años). The world according the GaWC. Loughborough University, Group of Analysis in World Cities. Recuperado de <https:/www.lboro.ac.uk/ gawc/gawcworlds.html>.

Glaeser, Edward L. (2011). Triumph of the city. Nueva York: Penguin Press.

Graham, Daniel y Spence, Nigel (1995). "Contemporary Deindustrialisation and Tertiarisation in the London Economy». Urban Studies, 32 (6), 885-911. <https://doi.org/10.1080/00420989550012708>

Hernández, E. Macarena y Usabiaga, Carlos (2016). Análisis del crecimiento económico y la convergencia: aplicaciones para las comunidades autónomas españolas. Cuadernos de Investigación 6. Sevilla: Consejería de Hacienda y Administración Pública, Junta de Andalucía. Recuperado de <https://www.juntadeandalucia.es/ export/drupaljda/publicacion/19/01/CuadernosInvestigacion_6.pdf>.

Knox, Paul L. y Taylor, Peter J. (eds.) (1995). World Cities in a World-System. Cambridge: Cambridge University Press.

Lois, Rubén y PiñeIra, María José (2017). «El modelo territorial y urbano resultante. Un sistema urbano metropolitano más formal que funcional y activo». En: FARINós, Joaquín y Olcina, Jorge (eds.). Geografía regional de España. Espacios y 
comunidades. Bases para una regionalización renovada del territorio español. Valencia: Tirant Humanidades, 427-507.

Markusen, Ann; Lee, Yoong-Sook y Di Giovanna, Sean (eds.) (1999). Second-Tier Cities: Rapid Growth Beyond the Metropolis. Minneapolis: University of Minnesota Press.

McCann, Philip y Acs, Zoltan J. (2011). "Globalization: Countries, cities and multinationals». Regional Studies, 45, 17-32. <https://doi.org/10.1080/00343404.2010.505915>

Meijers, Evert J. y Burger, Martijn J. (2015). «Stretching the concept of "borrowed size"». Urban Studies, 54, 269-291. <https://doi.org/10.1177/0042098015597642>

Meijers, Evert J.; Burger, Martijn J. y Hoogerbrugge, Marloes M. (2016). «Borrowing size in networks of cities: City size, network connectivity and metropolitan functions in Europe». Papers in Regional Science, 95, 181-198. $<$ https://doi.org/10.1111/pirs.12181>

MéndeZ, Ricardo (2006). «Las mutations des marchés métropolitaines du travail: realités et mythes dans la región de Madrid». En: Méndez, R. (ed.). Géographie de l'Espagne. París: L'Harmattan, 137-162.

- (2008). «Transformaciones del sistema productivo y nuevas formas metropolitanas. Una propuesta interpretativa». En: Ramírez, B. R. (coord.). Formas territoriales. Visiones y perspectivas desde la teoría. México: Universidad Autónoma Metropolitana, 175-205.

- (2013). «Economía del conocimiento y nuevos contrastes territoriales en España: una perspectiva multiescalar». Boletín de la Asociación de Geógrafos Españoles, 63, $7-32$. <https://doi.org/10.21138/bage.1604>

MÉndeZ, Ricardo y SÁNCHEZ, Simón (2010). «Spanish cities in the knowledge economy: Theoretical debates and empirical evidence». European Urban and Regional Studies, 18, 136-155. <https://doi.org/10.1177/0969776410381039>

Méndez, Ricardo; Michelini, Juan J.; Prada, José y TÉbar, Jesús (2012). «Economía creativa y desarrollo urbano en España: una aproximación a sus lógicas espaciales». Eure, 113, 5-32. <https://doi.org/10.4067/s0250-71612012000100001>

Musterd, Sako y Murie, Alana (2010). Making competitive cities. Pathways, actors and policies. Londres: Blackwell.

Musterd, Sako; Bontje, Marco y Rouwendal, Jan (eds.) (2016). Skills and cities. Oxon y Nueva York: Routledge-Regional Studies Association.

Ni, Pengfei y Kresl Peter (2010). The global urban competitiveness report - 2010. Cheltenham: Edward Elgar.

Pareja, Montserrat y Turmo, Joaquín (2013). «La necesaria transformación del modelo productivo en España: el papel del territorio». Documents d'Anàlisi Geogràfica, 59, 455-479. <https://doi.org/10.5565/rev/dag.59>

Parkinson, Michael; Meegan, Richard y Karecha, Jay (2015). "City Size and Economic Performance: Is Bigger Better, Small More Beautiful or Middling Marvellous?». European Planning Studies, 23, 1.054-1.068. <https://doi.org/10.1080/09654313.2014.904998>

Rozenblat, Céline (2007). «Les villes mediterranéennes dans la structuration du système des villes européennes». Treballs de la Societat Catalana de Geo- 
grafia, 63, 147-166. Recuperado de <https://publicacions.iec.cat/repository/ pdf/00000048/00000041.pdf>.

Rozenblat, Céline y Cicille, Patricia (2003). Les villes europeennes. Analyse comparative. Montpellier: Université de Montpellier III.

SÁnchez, Simón (2017). "The mobility of "creative workers" and their potential contribution to the recovery of old industrial cities in Spain». The Town Planning Review, 88, 83-107. $<$ https://doi.org/10.3828/tpr.2017.8>

Sánchez, Simón y Arellano, Alfonso (2010). "Aproximación a la economía del conocimiento en las ciudades españolas a través de la Muestra Continua de Vidas Laborales: aspectos metodológicos y avance de resultados». Colección de Estudios Económicos - FEDEA, 10, 1-42.

- (2012). «El empleo en la economía del conocimiento en España: características socioprofesionales y patrones de distribución territorial». Scripta Nova. Revista Electrónica de Geografía y de Ciencias Sociales, 399, 1-17.

SÁnchez, Simón; Méndez, Ricardo y Arellano, Alfonso (2014). «Creative economy and employment quality in large urban areas in Spain». Urban Geography, 35, 264-289. <https://doi.org/10.1080/02723638.2013.876145>

Sánchez Moral, Simón; Arellano, Alfonso y Díez, Roberto (2018). «Interregional mobility of talent in Spain: The role of job opportunities and qualities of places during the recent economic crisis». Environment and Planning A, 50 (4), 789-808.

<https://doi.org/10.1177/0308518x18761151>

Sassen, Saskia (1991). The Global City: New York, London, Tokyo. Princeton: Princeton University Press. $<$ https://doi.org/10.7440/res10.2001.14>

- (1994). Cities in a World Economy. Thousand Oaks: Pine Forge Press.

ScotT, Allen J. (1988). «Flexible production systems and regional development: The rise of new industrial spaces in North America and western Europe». International Journal of Urban and Regional Research, 12 (2), 171-186. <https://doi.org/10.1111/j.1468-2427.1988.tb00448.x>

- (2006). «Creative cities: conceptual issues and policy questions». Journal of Urban Affairs, 28, 1-17. $<$ https://doi.org/10.1111/j.0735-2166.2006.00256.x>

- (2012). A World in Emergence. Cities and regions in the 21st Century. Cheltenham, RU / Northampton, MA, EE. UU.: Edward Elgar.

Smith, David A. y Timberlake, Michael (1995). "Cities in global matrices: towards mapping the world-system's city system». En: KNox, Paul L. y TaYlor, Peter J. (eds.). World Cities in a World-System. Cambridge: Cambridge University Press, 79-97. <https://doi.org/10.1017/cbo9780511522192.006>

TaYlor, Peter J. (2001). "Specification of the World City Network». Geographical Analysis, 33, 181-194.

- (2003). World City Network: a global urban analysis. Londres: Routledge.

- (2012). "The Interlocking Network Model». En: Derudder, Ben; Hoyler, Michael; TAYlor, Peter J. y Witlox, Frank (eds.). International Handbook of Globalization and World Cities. Cheltenham: Edward Elgar, 51-63.

<https://doi.org/10.4337/9781781001011> 
Taylor, Peter J.; Catalano, Gilda, y Walker, David R. F. (2002). «Measurement of the World City Network». Urban Studies, 39, 2.367-2.376.

<https://doi.org/10.1080/00420980220080011>

Taylor, Peter J.; Hoyler, Michael y Sánchez, Simón (2015). «European Cities in Globalization: A Comparative Analysis Based on the Location Strategies of Advanced Producer Services». En: Cuadrado Roura, Juan R. Service industries and regions. Berlín y Heidelberg: Springer, 285-304.

<https://doi.org/10.1007/978-3-642-35801-2_12> 
